\title{
Comparative functional analysis of aquaporins/glyceroporins in mammals and anurans
}

\author{
Carissa M. Krane $\cdot$ David L. Goldstein
}

Received: 5 March 2007 / Accepted: 28 May 2007/Published online: 26 July 2007

(C) Springer Science+Business Media, LLC 2007

\begin{abstract}
Maintenance of fluid homeostasis is critical to establishing and maintaining normal physiology. The landmark discovery of membrane water channels (aquaporins; AQPs) ushered in a new area in osmoregulatory biology that has drawn from and contributed to diverse branches of biology, from molecular biology and genomics to systems biology and evolution, and from microbial and plant biology to animal and translational physiology. As a result, the study of AQPs provides a unique and integrated backdrop for exploring the relationships between genes and genome systems, the regulation of gene expression, and the physiologic consequences of genetic variation. The wide species distribution of AQP family members and the evolutionary conservation of the family indicate that the control of membrane water flux is a critical biological process. AQP function and regulation is proving to be central to many of the pathways involved in individual physiologic systems in both mammals and anurans. In mammals, AQPs are essential to normal secretory and absorptive functions of the eye, lung, salivary gland, sweat glands, gastrointestinal tract, and kidney. In urinary, respiratory, and gastrointestinal systems, AQPs are required for proper urine concentration, fluid reabsorption, and glandular secretions. In anurans, AQPs are important in mediating physiologic responses to changes in the external environment, including those that occur during metamorphosis and adaptation from an aquatic to terrestrial environment and thermal
\end{abstract}

C. M. Krane ( $\square)$

Department of Biology, University of Dayton, 300 College Park,

Dayton, Ohio 45469, USA

e-mail: Carissa.Krane@notes.udayton.edu

D. L. Goldstein

Department of Biological Sciences, Wright State University,

Dayton, Ohio 45435, USA acclimation in anticipation of freezing. Therefore, an understanding of AQP function and regulation is an important aspect of an integrated approach to basic biological research.

\section{Mechanisms of fluid homeostasis and aquaporins}

The maintenance of fluid homeostasis is critical to all life processes. Vertebrates have evolved intricate physiologic mechanisms for sensing and responding to changes in fluid composition and volume that are caused by environmental variables such as diet, health, hydration, injury, disease, temperature, and other stressors. The biological mechanisms that regulate fluid homeostasis in amphibians and mammals are complex and highly coordinated processes involving the precise regulation of ensembles of ion and water transporters. While osmotically driven transmembrane water movement can occur via simple diffusion through the lipid bilayer, selective membrane water permeability required for rapid and regulated physiologic processes such as secretion and reabsorption requires facilitation through proteinaceous water pores. Aquaporins (AQPs) are water-selective channels that function to increase plasma membrane water permeability in response to osmotic gradients. AQP1 (CHIP28), the first mammalian water channel to be functionally characterized, was isolated from the membranes of red blood cells as a $28-\mathrm{kDa}$ protein and was shown to increase membrane permeability in response to osmotic gradients (Preston and Agre 1991; Preston et al. 1992). Amino acid homology with the major intrinsic protein (MIP) of the lens indicated that AQP1 is a member of the MIP family of membrane proteins (Gorin 
et al. 1984). In 2003, Dr. Peter Agre was awarded the Nobel Prize in Chemistry to signify the importance of this discovery (Agre 2004). To date, more than 450 members of the MIP superfamily of integral membrane proteins have since been identified in a wide range of organisms, including mammals (reviewed in Krane and Kishore 2003), plants (reviewed in Chaumont et al. 2005), yeast (reviewed in Pettersson et al. 2005), bacteria (reviewed in Tanghe et al. 2006), and anurans (reviewed in Suzuki et al. 2007).

\section{Aquaporin structure}

MIP superfamily members are typically $28-30 \mathrm{kDa}$ in size and construct an integral membrane pore, characterized topographically by six transmembrane spanning domains, with cytosolic amino and carboxy termini (Fig. 1). Structural and amino acid similarities between the first and second half of the protein and comparative analysis of gene structure in paralogous sequences indicate that AQPs likely arose by way of an intragenic duplication that occurred relatively early in evolution (Pao et al. 1991). Intracellular loop B and extracellular loop E contain highly conserved asparagine-proline-alanine (NPA) motifs which are inserted into the membrane to create the functional water pore, generating what is referred to as the "hourglass model" (Jung et al. 1994). In some family members, a cysteine residue in the extracellular loop E (Cys 189 in human AQP1) that is situated close to the pore confers functional channel inhibition by mercurials through physical blockage of the

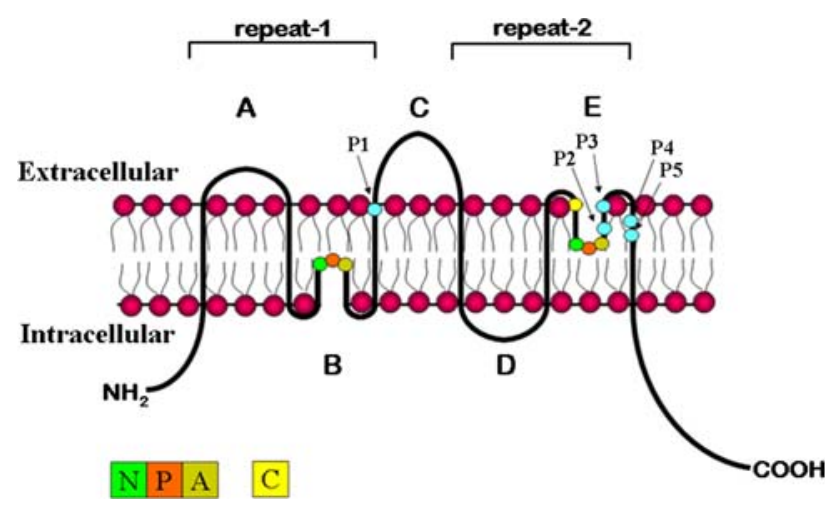

Fig. 1 Transmembrane structure of MIP family of integral membrane proteins. Hydropathy plots of primary amino acid sequence of the major intrinsic membrane protein family identified a sixtransmembrane-spanning topology. X-ray crystallography and electron microscopy confirmed this structure and provided further proof of homotetramer membrane assembly. The conserved NPA motifs are indicated in loops B and E. The cysteine shown in loop E confers mercury sensitivity in many MIP proteins. Amino acid positions P1$\mathrm{P} 5$ confer functional permeability for either water or glycerol in the AQP and GLP subfamilies, respectively pore (Preston et al. 1993; Zhang et al. 1993). Recent studies have indicated that the water permeability of a subset of aquaporins can be blocked by quaternary ammonium compounds such as tetraethyl ammonium (TEA) (Detmers et al. 2006). AQPs assemble as homotetramers in the membrane; however, each monomer is a functional water pore that supports bidirectional, osmotically driven transmembrane water flow. In vivo, the direction of water flow through AQPs is determined by the osmotic gradient that exists across the membrane during specific physiologic processes such as absorption or secretion driven by active ion transport.

The NPA motifs are present in nearly all MIP family members, with a few exceptions. Among the 13 known mammalian MIP family members, AQP7, AQP11, and AQP12 encode variant versions of the NPA box in loop B, and AQP7 also has a variant second NPA box in loop E. The proline in the NPA box of loop B of AQP7 is changed to an alanine, thereby changing the first NPA motif to NAA, whereas a serine replaces the alanine in loop E, resulting in an NPS motif (reviewed in Zardoya 2005). The first NPA box in the B loops of AQP 11 and AQP12 is changed to NPC and NPT, respectively (Gorelick et al. 2006; Itoh et al. 2005).

\section{Aquaporins vs. aquaglyceroporins}

\section{Aquaporins}

Other primary amino acid sequence differences that exist between members of the MIP family have given rise to two structural classes of proteins whose functions are distinctive. Aquaporins (AQPs) are water-selective members of the MIP family, whereas aquaglyceroporins (GLPs) transport both water and organic compounds such as glycerol, urea (reviewed in Hara-Chikuma and Verkman 2006), and potentially other small solutes (e.g., $\mathrm{NH}_{3}$ and $\mathrm{NH}_{4}^{+}$; Holm et al. 2005). The determination of pore selectivity for water in the AQP subclass has been examined through a variety of experimental methods, including site-directed mutagenesis, chimeric domain swaps, membrane permeability assays, electron crystallography, X-ray crystallography, and molecular dynamic simulations (reviewed in Gonen and Walz 2006). X-ray crystallographic analysis of bovine AQP1 from red blood cells at a $2.2-\AA$ resolution identified extracellular and cytosolic pore entry/exit passageways for water molecules that are separated by a central constriction region of the channel (Sui et al. 2001). The constriction region is formed by the interactions of four amino acids within the pore (His 182, Arg 197, Cys 191, Phe 58), which limits the pore size to a $2.8-\AA$ diameter (Sui et al. 2001). Three of the amino acids found within the constriction site are 
Table 1 Amino acid conservation at sites determinative of AQP vs. GLP selectivity

\begin{tabular}{|c|c|c|c|c|c|c|}
\hline Position & Location & $\mathrm{AQP}$ & GLP & HC-1 & $\mathrm{HC}-2$ & HC-3 \\
\hline $\mathrm{P} 1$ & Transmembrane Helix 3 & Nonaromatic & Aromatic & Thr & Thr & Tyr \\
\hline $\mathrm{P} 2$ & Extracellular Loop E & Small and uncharged & Asp & Ser & Ser & Asp \\
\hline $\mathrm{P} 3$ & Extracellular Loop E & Small and uncharged & Lys or Arg & Ala & Ala & Arg \\
\hline $\mathrm{P} 4$ & Transmembrane Helix 6 & Aromatic & Pro & Phe & Phe & Pro \\
\hline P5 & Transmembrane Helix 6 & Aromatic & Nonaromatic & $\operatorname{Trp}$ & Trp & Iso \\
\hline
\end{tabular}

Amino acids at positions P1-P5 determine AQP vs. GLP function and are conserved in mammalian and nonmammalian species (Froger et al. 1998; Heymann and Engel 2000; Lagree et al. 1999). Amino acids at positions P1-P5 for AQP members HC-1, HC-2, and HC-3, the latter a glyceroporin, from the anuran $H$. chrysoscelis $(\mathrm{HC})$ are included as examples of conservation in a nonmammalian species (Zimmerman et al. 2007).

conserved in all water-specific MIP members (Arg 197, His 182, Phe 58; Park and Saier 1996) and contribute to the water selectivity seen in the AQP subclass of the MIP family. In addition, a "selectivity filter," consisting of six amino acids, was identified. It forces water to make and break hydrogen bonds as molecules pass single file through the pore (Sui et al. 2001). Using real-time molecular dynamic simulations of water movement through human AQP1, De Groot and Grubmüller (2001) proposed a two-stage filter model in which the NPA motif forms a selectivity-determining region, and a second region termed the aromatic/arginine $(\mathrm{ar} / \mathrm{R})$ region functions as a proton filter.

\section{Aquaglyceroporins}

Transport of glycerol has been studied in a few cell types, vertebrate and otherwise. In some cells glycerol crosses the membrane readily, whereas others are quite impermeable (Vom Dahl and Häussinger 1997). In liver, permeation of glycerol across the membrane (as opposed to its phosphorylation and metabolism) is the rate-limiting step in glycerol utilization ( $\mathrm{Li}$ and Lin 1983). Characteristics described for this transport include a combination of simple diffusion and $\mathrm{H}^{+}$- or $\mathrm{Na}^{+}$-coupled cotransport (Carlsen and Wieth 1976; Lages and Lucas 1995; Lucas et al. 1990). The mechanism is distinct from the glucose transporter and may be phloretin-sensitive (vom Dahl and Häussinger 1997). These studies may well have incorporated characteristics of multiple transport mechanisms, which remain poorly defined. One mechanism that is common to cells ranging from E. coli (Heller et al. 1980) to insect (Farinas et al. 1995) to mammalian kidney involves glycerol transport via proteins from the aquaporin family. The "selectivity" of the AQP subclass was determined based on comparisons made with members of the GLP subclass. The best studied member of the GLP subclass is $G l p F$, a glycerol facilitator isolated from $E$. coli that is permeable to glycerol, urea, and glycine, with very low water permeability (Borgnia and Agre 2001; Maurel et al. 1994). Although GlpF assembles a transmembrane structure that is roughly sim- ilar to AQP1, the channel is asymmetric, and the lengths of the extracellular loops and constituents at several amino acid positions within the constriction region and selectivity filter differ significantly from AQP1 (Lu et al. 2003; Sui et al. 2001; reviewed in Gonen and Walz 2006). Five amino acid positions (P1-P5) located in transmembrane helix 3 (P1), extracellular loop $\mathrm{E}(\mathrm{P} 2, \mathrm{P} 3)$, and transmembrane helix 6 (P4, P5) differ consistently between the AQP and GLP subfamilies in both mammalian and nonmammalian species (Table 1; Fig. 1) (Froger et al. 1998; Heymann and Engel 2000; Lagree et al. 1999). X-ray crystallographic images in the absence of glycerol confirmed the results of the functional studies showing that GlpF does facilitate water transport (Sui et al. 2001; Tajkhorshid et al. 2002), although GlpF-mediated water permeability is far less than glycerol permeability. Since then the structure of several members of both the AQP and GLP subfamilies in a variety of organisms has been resolved at the atomic level (reviewed in Gonen and Walz 2006).

\section{Functional and phylogenetic classes of mammalian aquaporins/aquaglyceroporins}

Thirteen functionally and phylogenetically distinct mammalian water channels (AQP0-AQP12) have been identified on the basis of sequence homology to AQP1. Evolutionary comparison of mammalian MIP sequences classify AQP0, AQP1, AQP2, AQP4, AQP5, AQP6, and AQP8 as members of the water-selective aquaporin subgroup, whereas AQP3, AQP7, AQP9, and AQP10 are evolutionarily grouped as aquaglyceroporins (Gonen and Walz 2006; Gorelick et al. 2006; Zardoya 2005) (Fig. 2, Table 2). AQP11 and AQP12 are the most distantly related paralogs (Morishita et al. 2005). They have only approximately $20 \%$ homology with the MIP family and may constitute a third functionally distinct evolutionary branch of the MIP superfamily (Gorelick et al. 2006; Itoh et al. 2005; Morishita et al. 2004, 2005). 


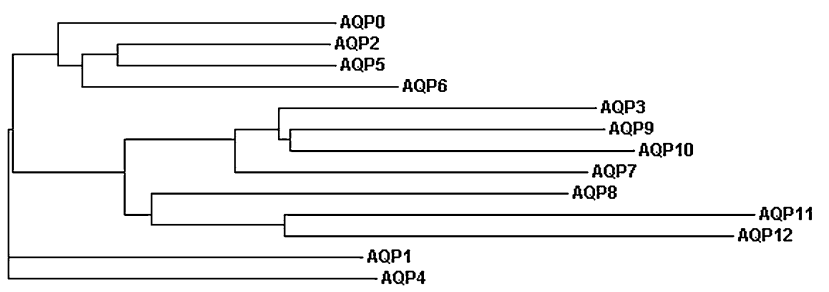

Fig. 2 Phylogram of human AQP/GLPs. A phylogram was generated using human AQP/GLP protein sequences available through NCBI/ Swiss-PROT and the multisequence alignment program ClustalW available at http://www.ebi.ac.uk/clustalw/index.html. The phylogenetic relationships shown here are consistent with previously published trees (Gorelick et al. 2006; Itoh et al. 2005)

Typically, MIP family members are functionally characterized for osmotic water permeability by expressing the candidate AQP/GLP cRNA in Xenopus oocytes and assessing cell volume changes upon hypotonic challenge (Preston et al. 1992). Likewise, solute (i.e., urea/glycerol) permeability can be determined by measuring solute uptake in isotonic solution. In some instances, AQP/GLP function has also been assessed in reconstituted proteoliposomes (Zeidel et al. 1992) or by expression in yeast (Lagree et al. 1998). Functionally, AQPs 0-10 support various levels of transmembrane water permeability (Table 2). AQP0, AQP6, AQP9, and AQP10 show low water permeability compared with AQP1, AQP2, AQP3, AQP4, AQP5, AQP7, and AQP8. The water permeability of AQP3 and AQP6 is affected by $\mathrm{pH}$. AQP3, AQP7, AQP9, and AQP10 are also permeable to both urea and glycerol, whereas AQP8 has been reported to exhibit urea and ammonia permeability (Saparov et al. 2007). Interestingly, both AQP7 and AQP9 have been reported to facilitate arsenite uptake (Liu et al. 2002), and AQP6 functions as an anion channel, with permeability to nitrate and chloride (Ikeda et al. 2002; Yasui et al. 1999). The membrane transport properties of AQP11 and AQP12 are currently unknown. Evaluation of AQP11 function in Xenopus oocytes failed to identify any water, urea, glycerol, or ion permeability under various $\mathrm{pH}$ conditions (Gorelick et al. 2006). The transport properties of AQP12 remain unstudied because of the inability to obtain adequate plasma membrane expression in Xenopus oocytes (Itoh et al. 2005).

\section{Mammalian AQP/GLP expression}

The subsets of organ, tissue, cellular, and subcellular expression patterns of the 13 known mammalian MIP family members are unique for each protein (Table 2). For example, whereas AQP1 is constitutively expressed in diverse tissues, $\mathrm{AQP} 2, \mathrm{AQP10}$, and $\mathrm{AQP12}$ show a narrow

Table 2 Phylogenetic/functional classifications and tissue distribution of mammalian MIPs

\begin{tabular}{|c|c|c|c|}
\hline $\begin{array}{l}\text { Gene } \\
\text { name }\end{array}$ & $\begin{array}{l}\text { Phylogenetic } \\
\text { AQP/GLP }\end{array}$ & $\begin{array}{l}\text { Functional } \\
\text { permeability }\end{array}$ & Tissue/cellular localization \\
\hline AQP0 & AQP & Water & Lens of the eye \\
\hline AQP1 & AQP & Water & $\begin{array}{l}\text { Kidney (proximal tubule and thin descending limb of the loop of Henle), erythrocytes, } \\
\text { capillary endothelium, choroid plexus, corneal epithelium, ear, lung, GI tract, skeletal } \\
\text { muscle, heart muscle }\end{array}$ \\
\hline AQP2 & $\mathrm{AQP}$ & Water & $\begin{array}{l}\text { Kidney (principal cells of the collecting duct and connecting tubules; apical surface and } \\
\text { subapical vesicles) }\end{array}$ \\
\hline AQP3 & GLP & $\begin{array}{l}\text { Urea and glycerol; } \\
\text { water }\end{array}$ & $\begin{array}{l}\text { Kidney (principal cells of the collecting duct and connecting tubules; basolateral surface), } \\
\text { airways, lung, GI tract, brain, ear, urinary bladder, cornea, epidermis }\end{array}$ \\
\hline AQP4 & AQP & Water & $\begin{array}{l}\text { Kidney (collecting duct principal cells; basolateral), retina, ear, airways, lung, GI tract, fast- } \\
\text { twitch skeletal muscle, glial cells at blood brain barrier, astrocytes }\end{array}$ \\
\hline AQP5 & $\mathrm{AQP}$ & Water & $\begin{array}{l}\text { Salivary gland, lacrimal gland, trachea, epithelia of nasopharynx and airways, alveolar type } 1 \\
\text { cells, ear, eye, placenta, pancreas }\end{array}$ \\
\hline AQP6 & $\mathrm{AQP}$ & $\begin{array}{l}\text { Anions }\left(\mathrm{NO}_{3}^{-} \text {and } \mathrm{Cl}^{-} \text {; }\right. \\
\text { water }\end{array}$ & Kidney (intracellular vesicles in type A intercalated cells of the collecting duct) \\
\hline AQP7 & GLP & $\begin{array}{l}\text { Urea and glycerol; } \\
\text { water, arsenite }\end{array}$ & Testis, sperm, kidney (proximal tubule), adipose tissue, skeletal muscle \\
\hline AQP8 & AQP & Urea and $\mathrm{NH}_{3}$, water & $\begin{array}{l}\text { Testis, sperm, GI tract, placenta, kidney (proximal tubule and collecting duct), airways, liver } \\
\text { salivary glands, glial and neuronal cells, pancreas }\end{array}$ \\
\hline AQP9 & GLP & $\begin{array}{l}\text { Urea and glycerol; } \\
\text { water, arsenite }\end{array}$ & $\begin{array}{l}\text { Liver, testis, sperm, spleen, brain, leukocytes, kidney, lung, brain (astrocytes and ependyma } \\
\text { cells) }\end{array}$ \\
\hline AQP10 & GLP & $\begin{array}{l}\text { Urea and glycerol; } \\
\text { water }\end{array}$ & Duodenum, jejunum \\
\hline AQP11 & *SuperAQP & Unknown & Kidney (intracellular localization in proximal tubule), liver, testis, brain \\
\hline AQP12 & *SuperAQP & Unknown & Pancreas (acinar cells) \\
\hline
\end{tabular}


range of tissue-specific expression: AQP2 is predominantly present in principal cells of the renal collecting duct, with minor functionally significant expression in epididymis (Nelson et al. 1998) and inner ear (Merves et al. 2000), AQP10 in the small intestine, and AQP12 in the pancreas (Table 2). Moreover, most if not all MIP genes are subject to temporal and tissue-selective expression via mechanisms that control transcription and translation, and in some cases through post-translational modifications, vesicular trafficking, and polar membrane insertion. AQP2 is perhaps the best-studied MIP with regard to mechanisms that regulate its expression. In the renal collecting duct, AQP2 vesicular trafficking is regulated by arginine vasopressin (AVP), the mammalian antidiuretic hormone (ADH), in response to dehydration (Kishore et al. 1996; Nielsen et al. 1995). In addition, AQP2 mRNA and protein expression are upregulated in response to chronic dehydration (DiGiovanni et al. 1994; Terris et al. 1996). Other signaling cues responsible for altering MIP gene expression include cAMP (Yang et al. 2003), cGMP (Ishikawa et al. 1998, 2002) NO (Ishikawa et al. 2002; Nagai et al. 2007), hypertonicity (Hoffert et al. 2000), cholinergic stimulation (Ishida et al. 1997), TNF- $\alpha$ (Towne et al. 2001), and steroids (King et al. 1996). It is clear that MIP family gene expression is highly regulated at multiple levels in response to a variety of physiologic triggers. The list of regulators will no doubt continue to expand as more is learned about the importance of MIP function during development and in normal physiologic and pathophysiologic states.

\section{Physiologic function of AQP/GLPs in mammals}

Some of what is known of the physiologic function of AQPs has been learned by examining the clinical manifestation and associated pathology of AQP deficiency in human disease. Human disorders whose pathogeneses are associated with defects in water channel proteins include inherited cataracts (AQP0; Berry et al. 2000; Francis et al. 2000) and nephrogenic diabetes insipidus (AQP2; Deen et al. 1994; reviewed in Knoers and Deen 2001), and water channel dysfunctions have been implicated in the etiology of Sjogren's syndrome (AQP5; Beroukas et al. 2001; Steinfeld et al. 2001; Tsubota et al. 2001). Individuals lacking functional AQP1 have a decrease in pulmonary vascular permeability (King et al. 2002) and fail to concentrate urine maximally when dehydrated (King et al. 2001). The list of AQP involvement in human disease is likely to increase as an understanding of the complexity of AQP function becomes better realized.

Targeted disruptions of individual water channel proteins in knockout mouse models have been useful in further elucidating the role of AQP/GLPs in whole-animal physiology. Table 3 summarizes the phenotypes reported to date for the AQP-deficient mice currently available. The information gleaned from the knockout strains implicates abnormal AQP function/regulation as a potential contributor to other urine-concentrating defects (AQP3; Ma et al. $2000 \mathrm{~b}$ ) and to human diseases such as disorders of the skin (AQP3; Ma et al. 2002), impaired wound healing (AQP3; Hara et al. 2002), hearing loss (AQP4; Li and Verkman 2001), salivary gland secretory defects (Ma et al. 1999; Krane et al. 2001b), impaired sweat gland function (Nejsum et al. 2002), asthma (AQP5; Krane et al. 2001a), diabetes and insulin resistance (AQP7; Hibuse et al. 2005), obesity (AQP7; Hara-Chikuma et al. 2005) and polycystic kidney disease (AQP11; Morishita et al. 2005). As such associations emerge for human pathologies, designed pharmacologic inhibition of AQP function may be of specific clinical utility, such as for preventing cellular migration during metastatic tumor progression (AQP1; Saadoun et al. 2005) or in response to brain edema or ischemic injury (AQP4; Manley et al. 2000).

\section{AQPs/GLPs in thermal tolerance: the importance of comparative analyses of nonmammalian subjects}

Aquaporin proteins are found ubiquitously among the kingdoms of living things. Many of these organisms experience extremes of thermal and osmotic stress far beyond those tolerated by mammals. One such circumstance is the possibility of substantial cellular dehydration elicited by freezing; organisms ranging from bacteria through certain vertebrates tolerate subfreezing temperatures, and they do so using a combination of water and solute transport mechanisms.

Recently, the importance of AQP/GLP in the process of glycerol-facilitated cryopreservation has been shown under natural and experimental conditions. Glycerol is an organic solute commonly used in biomedicine as a cryoprotectant to enable bacterial, fungal, and embryonic cells to freeze at ultralow temperatures without compromised viability. Enhanced AQP/GLP expression correlates with improved freeze tolerance in baker's yeast (Tanghe et al. 2002) and sperm (Dibas et al. 1998), and artificial AQP/GLP expression improves viability following cryopreservation of fish embryos (Hagedorn et al. 2002) and mouse oocytes (Edashige et al. 2003), suggesting that facilitated glycerol transport through AQP/ GLP may participate in the physiology of freeze tolerance in animals. Clinical cryopreservation is currently most successful with small or single-celled tissues. Therefore, insights into how a multicellular organism survives freezing could yield important clues to the cryopreservation of larger tissues and organs. For exam- 
Table 3 Phenotypes of MIP-deficient mouse strains

\begin{tabular}{|c|c|c|}
\hline Gene Name & Phenotype of MIP-deficient mouse strains & Reference \\
\hline AQP0 & Cataracts & Shiels and Bassnett 1996 \\
\hline \multirow[t]{2}{*}{ AQP1 } & Polyuria, defective proximal tubule fluid absorption & Ma et al. 1998 \\
\hline & Decreased osmotic water permeability across endothelium & Bai et al. 1999 \\
\hline $\mathrm{AQP} 2$ & Severe polyuria; failure to thrive & Rojek et al. 2006 \\
\hline \multirow[t]{3}{*}{ AQP3 } & Urinary concentrating defect-NDI & Ma et al. $2000 \mathrm{~b}$ \\
\hline & Reduced skin hydration and elasticity & Ma et al. 2002 \\
\hline & Delayed wound healing & Hara et al. 2002 \\
\hline \multirow[t]{3}{*}{ AQP4 } & Mild urine-concentrating defect & Ma et al. 1997 \\
\hline & Reduced injury-induced brain edema & Manley et al. 2000 \\
\hline & Hearing defects & Li and Verkman 2001 \\
\hline \multirow[t]{5}{*}{ AQP5 } & Impaired salivary secretion & Krane et al. 2001b; Ma et al. 1999 \\
\hline & Airway hyperresponsiveness to cholinergic stimulation & Krane et al. 2001a \\
\hline & Impaired stimulated sweat secretion & Nejsum et al. 2002 \\
\hline & Decreased osmotic water permeability across alveolar epithelium & Ma et al. 2000a \\
\hline & Impaired secretion in airway submucosal glands & Song and Verkman 2001 \\
\hline AQP6 & Unknown & \\
\hline \multirow[t]{2}{*}{ AQP7 } & Increased body fat with adipocyte hypertrophy & Hara-Chikuma et al. 2005 \\
\hline & Increased body weight and age-dependent insulin resistance & Hibuse et al. 2005 \\
\hline AQP8 & Mild hypertriglyceridemia & Yang et al. 2005 \\
\hline AQP9 & Unknown & \\
\hline AQP10 & Unknown & \\
\hline AQP11 & Polycystic kidney disease (proximal tubule) & Morishita et al. 2005 \\
\hline AQP12 & Unknown & \\
\hline
\end{tabular}

ple, modeling studies have suggested that cryopreservation of whole mammalian kidneys, which would entail perfusion of cryopreservative solution such as glycerol through the vasculature, would succeed best if tissue permeability to the cryopreservative agent were high, thereby minimizing osmotically induced changes in cellular and extracellular volumes (Lachenbruch et al. 1998). Thus, the comparative analysis of AQPs/GLPs in amphibians, a subset of which undergo a physiologic process of cryopreservation and freeze tolerance (sometimes involving glycerol accumulation), may serve as a model for testing such ideas.

\section{Role of the kidneys in conserving organic solutes in anurans}

Renal conservation of cryoprotective solutes may be critical to freeze-tolerant anurans. Repeated cycles of freezing and thawing deplete glycogen stores in anuran liver (Lee and Costanzo 1993), quite likely because glucose is lost in the urine: The renal tubules have a limited capacity for glucose reabsorption, and this may be overwhelmed at high glucose concentrations occurring after freezing (Layne et al. 1996). Wood frogs may compensate for this loss by cutaneous uptake of excreted glucose.

In wood frogs, glycogenesis is initiated promptly upon thawing, thereby minimizing the duration of high plasma glucose and so its potential urinary loss. In contrast, gray treefrogs retain high plasma glycerol concentrations for weeks, before and after freezing. How do they avoid urinary loss of this solute? Presumably glycerol is filtered, albeit at a reduced rate, in cold-acclimated frogs, which have reduced rates of glomerular filtration (Zimmerman et al. 2007). Thus, reabsorption of filtered glycerol should be at a premium.

In other circumstances when glomerular filtration rate (GFR) is reduced in amphibians, plasma arginine vasotocin (AVT), the amphibian antidiuretic hormone, is elevated (Nouwen and Kuhn 1983; Rosenbloom and Fisher 1974). AVT may act on the renal vasculature to reduce GFR (Pang 1983), on the bladder to increase water permeability, and on the renal tubules (Uchiyama 1994). The physiologic response of the latter structures to AVT is not known, but one response of vertebrate kidneys to $\mathrm{ADH}$ is upregulation and membrane insertion of aquaporins, which could mediate reabsorption of water and/or glycerol. 


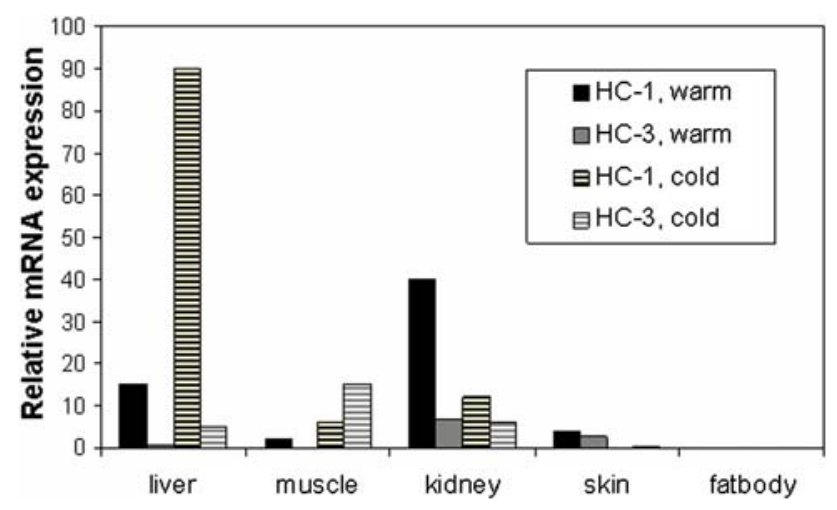

Fig. 3 Relative HC-1 and HC-3 mRNA expression in warm- vs. cold-acclimated tissues from $H$. chrysoscelis. Relative mRNA expression (real-time PCR, expression of HC-1 or HC-3 mRNA normalized to expression of $\beta$-actin mRNA) in an aquaporin (HC-1) and a glyceroporin (HC-3) from the anuran Hyla chrysoscelis. Note that expression varies both among tissues and depending on acclimation to either warm $\left(20^{\circ} \mathrm{C}\right)$ or cold $\left(4^{\circ} \mathrm{C}\right)$ conditions

\section{Aquaporins in amphibians}

Historically, amphibians have played a critical role in the conceptualization of and suggestion for the existence of water channels long before the first water channel was cloned. Indeed, even before aquaporins were understood as such, careful study of toad urinary bladder suggested the "shuttle hypothesis," based on visualization of "particle aggregates", that appeared to be inserted into and retrieved from the apical membrane under conditions of changing water reabsorption (e.g., Wade 1989; Wade et al. 1981). Additional evidence has confirmed a role for AQPs and GLPs in amphibian osmoregulation. Aquaporins have been identified in amphibian skin, bladder, fat body, and elsewhere (Ma et al. 1996; Virkki et al. 2002; Zimmerman et al. 2007), and several proteins of the aquaporin family have been sequenced from anurans. Phylogenetic analysis of 17 anuran AQP mRNA sequences deposited in public databases has revealed six classes of anuran AQPs, two of which are distinct to anurans (reviewed in Suzuki et al. 2007). "FA-CHIP", in Rana esculenta (Abrami et al. 1994), "AQP-t1"' in Bufo marinus (Ma et al. 1996), AQPh1 in Hyla japonica (Hasegawa et al. 2003), and HC-1 in Hyla chrysoscelis (Zimmerman et al. 2007) resemble each other in both sequence and wide tissue distribution patterns. These proteins are also similar to mammalian AQP1 (76\%-98\% sequence identity), and expression cloning has confirmed that AQP-t1, AQP-h1, and HC-1 function as water but not as glycerol channels (Hasegawa et al. 2003; Ma et al. 1996; Zimmerman et al. 2007) (Table 1). Temperature-sensitive regulation of $\mathrm{HC}-1$ expression was seen in brain, kidney, and liver; frogs acclimated to cold conditions $\left(4^{\circ} \mathrm{C}\right)$ had higher $\mathrm{HC}-1$ mRNA expression in the liver than did warm-acclimated frogs, whereas in brain and kidney warm-acclimated frogs expressed higher levels of HC-1 (Zimmerman et al. 2007) (Fig. 3). A second AQP, $\mathrm{HC}-2$, has recently been isolated from urinary bladder cDNA from $H$. chrysoscelis and shows strong amino conservation compared with mammalian AQP2 (69\% identity, 85\% similarity; Zimmerman et al. 2007). Like hAQP2, HC-2 was functionally determined to be within the AQP subclass, and it supported osmotically driven water transport (Zimmerman et al. 2007) (Table 1). HC-2 mRNA was detected primarily in organs of osmoregulation (skin, bladder, and kidney; Zimmerman et al. 2007). Interestingly, like HC-1, HC-2 expression varied depending on thermal conditions, i.e., hydrated frogs that were acclimated to cold conditions $\left(4^{\circ} \mathrm{C}\right)$ had high levels of $\mathrm{HC}-2$ expression in skin, whereas no HC-2 expression was observed from the ventral skin of hydrated warm-acclimated frogs (Zimmerman et al. 2007).

To date, four anuran sequences similar to mammalian AQP3 have been identified. They include AQP3 from $X$. laevis (Schreiber et al 2000), AQP from X. tropicalis (unpublished; GenBank Accession number CR855446), AQP-h3BL from $H$. japonica (Akabane et al. 2007), and HC-3 from H. chrysoscelis (Zimmerman et al. 2007). HC-3 from $H$. chrysoscelis shows $82 \%$ identity and $94 \%$ amino acid similarity with mammalian AQP3, and functionally it performs as a GLP, with low water permeability and high glycerol permeability (Zimmerman et al. 2007) (Table 1). HC-3 mRNA exhibited both tissue-specific and thermalselective patterns of expression. Of special note, tissue glycerol concentrations increased in the liver and skeletal muscle in cold-acclimated frogs compared with warmacclimated frogs (Fig. 3). The increase in glycerol concentration in these tissues corresponds well with an increase in HC-3 mRNA abundance in muscle, liver, and bladder in cold-acclimated frogs (Zimmerman et al. 2007). Studies of mammalian GLPs are just beginning to elucidate potential physiologic roles for their facilitation of glycerol transport. These roles include glycerol export from adipocytes (Hara-Chikuma et al. 2005) and a contribution to pliability in skin (Ma et al. 2002). Amphibians that naturally accumulate glycerol represent a natural model for studying the roles and regulation of glycerol-transporting aquaporins.

Two aquaporins from $H$. japonica (AQP-h2 and AQPh3) have been sequenced (Hasegawa et al. 2003; Tanii et al. 2002) that have high homology to each other and to AQP-t2 and AQP-t3 from B. marinus. Suzuki et al. (2007) have suggested that these four genes form an anuran-specific, phylogenetically distinct MIP subclass (type AQPa2). AQP-h2 and AQP-h3 are both expressed in ventral skin, whereas AQP-h2 is also expressed in the urinary bladder. Expression of both is upregulated by AVT (Hasegawa et al. 2005). Coexpression of the AVT receptor, AQP-h2, and 
AQP-h3 during metamorphosis, when the animals are undergoing a transition from aquatic to terrestrial environment, suggests a role for AVT-regulated AQPs in this process (Hasegawa et al. 2004). A second anuran-specific phylogenetic class, type-a1, has been assigned for a novel aquaporin identified from oocytes of $X$. laevis; that protein exhibits unique mercury sensitivity and less than $50 \%$ amino acid identity to the most closely related mammalian aquaporins (Virkki et al. 2002).

\section{Conclusion}

Studies of mammalian aquaporins have revealed many details of structure and function and are beginning to yield insights into pathogenic mechanisms. Nevertheless, mammalian aquaporins comprise a relatively limited subset of proteins from this large class of molecules. Proteins from the MIP family are present in every sort of organism, from bacteria through fungi, plants, and animals. It is likely that novel insights into structure-function relationships, into mechanisms of regulation, and into physiologic roles will derive from this diversity of organisms. Amphibians present particularly attractive models for studies of aquaporin function in vertebrate osmoregulation and thermoregulation; transitions from water to land and tolerance of tissue freezing are the epitomes of combined osmotic and thermal demands. Thus, just as studies of amphibians contributed to the original elucidation of aquaporin function, before we knew of aquaporins per se, we suggest that such studies will continue to contribute to a fundamental understanding of these ubiquitous proteins.

Acknowledgments The authors thank Dr. B.K. Kishore for reviewing the manuscript. This work was supported in part by research grant NSF IOB-0517301 to DLG and CMK.

\section{References}

Abrami L, Simon M, Rousselet G, Berthonaud V, Buhler J-M, et al. (1994) Sequence and functional expression of an amphibian water channel, FA-CHIP: a new member of the MIP family. Biochim Biophys Acta 1192:147-151

Agre P (2004) Aquaporin water channels (Nobel lecture). Angew Chem Int Ed Engl 43:4278-4290

Akabane G, Ogushi Y, Hasegawa T, Suzuki M, Tanaka S (2007) Gene cloning and expression of an aquaporin, AQP-h3BL, in the basolateral membrane of water-permeable epithelial cells in osmoregulatory organs of the tree frog. Am J Physiol Regul Integr Comp Physiol 292:R2340-2351

Bai C, Fukuda N, Song Y, Ma T, Matthay MA, et al. (1999) Lung fluid transport in aquaporin-1 and aquaporin-4 knockout mice. $\mathrm{J}$ Clin Invest 103:555-561

Beroukas D, Hiscock J, Jonsson R, Waterman SA, Gordon TP (2001) Subcellular distribution of aquaporin 5 in salivary glands from Sjogren's syndrome. Lancet 358:1875-1877
Berry V, Francis P, Kaushal S, Moore A, Bhattacharya S (2000) Missense mutations in MIP underlie autosomal dominant polymorphic and lamellar cataracts linked to $12 \mathrm{q}$. Nat Genet 25:15-17

Borgnia MJ, Agre P (2001) Reconstitution and functional comparison of purified GlpF and AqpZ, the glycerol and water channels from Escherichia coli. Proc Natl Acad Sci U S A. 98:2888-2893

Carlsen A, Wieth JO (1976) Glycerol transport in human red cells. Acta Phys Scand 97:501-513

Chaumont F, Moshelion M, Daniels MJ (2005) Regulation of plant aquaporin activity. Biol Cell 97:749-764

De Groot BL, Grubmüller H (2001) Water permeation across biological membranes: mechanism and dynamics of aquaporin1 and GlpF. Science 294:2353-2357

Deen PM, Verdijk MA, Knoers NV, Wieringa B, Monnens LA, et al. (1994) Requirement of human renal water channel aquaporin-2 for vasopressin-dependent concentration of urine. Science 264:92-95

Detmers FJM, de Groot BL, Müller EM, Hinton A, Konings IBM, et al. (2006) Quaternary ammonium compounds as water channel blockers. J Biol Chem 281:14207-14214

Dibas AI, Mia AJ, Yorio T (1998) Aquaporins (water channels): role in vasopressin-activated water transport. Proc Soc Exp Biol Med 219:183-199

DiGiovanni SR, Nielsen S, Christensen EI, Knepper MA (1994) Regulation of collecting duct water channel expression by vasopressin in Brattleboro rat. Proc Natl Acad Sci U S A 91:8984-8988

Edashige K, Yamaji Y, Kleinhans FW, Kasai M (2003) Artificial expression of aquaporin-3 improves the survival of mouse oocytes after cryopreservation. Biol Reprod 68:87-94

Farinas J, Simanek V, Verkman AS (1995) Cell volume measured by total internal reflection microfluorimetry: application to water and solute transport in cells transfected with water channel homologs. Biophys J 68:1613-1620

Francis P, Chung JJ, Yasui M, Berry V, Moore A, et al. (2000) Functional impairment of lens aquaporin in two families with dominantly inherited cataracts. Hum Mol Genet 9:2329-2334

Froger A, Tallur B, Thomas D, Delamarche C (1998) Prediction of functional residues in water channels and related proteins. Protein Sci 7:1458-1468

Gonen T, Walz T (2006) The structure of aquaporins. Q Rev Biophys 39:361-396

Gorelick DA, Praetorius J, Tsunerai T, Nielsen S, Agre P (2006) Aquaporin-11: A channel protein lacking apparent transport function expressed in brain. BMC Biochem 7:1-14

Gorin MB, Yancey SB, Cline J, Revel JP, Horwitz J (1984) The major intrinsic protein (MIP) of the bovine lens fiber membrane: characterization and structure based on cDNA cloning. Cell 39:49-59

Hagedorn M, Lance SL, Fonseca DM, Kleinhans FW (2002) Altering fish embryos with aquaporin-3: an essential step toward successful cryopreservation. Biol Reprod 67:961-966

Hara M, Ma T, Verkman AS (2002) Selectively reduced glycerol in skin of aquaporin-3 deficient mice may account for impaired skin hydration, elasticity and barrier recovery. J Biol Chem 277:46616-46621

Hara-Chikuma M, Verkman AS (2006) Physiological roles of glycerol-transporting aquaporins: the aquaglyceroporins. Cell Mol Life Sci 63:1386-1392

Hara-Chikuma M, Sohara E, Rai T, Ikawa M, Okabe M, et al. (2005) Progressive adipocyte hypertrophy in aquaporin 7 deficient mice: adipocyte glycerol permeability as a novel regulator of fat accumulation. J Biol Chem 280:15493-15496

Hasegawa T, Tanii H, Suzuki M, Tanaka S (2003) Regulation of water absorption in the frog skins by two vasotocin-dependent 
water-channel aquaporins, AQP-h2 and AQP-h3. Endocrinology 144:4087-4096

Hasegawa T, Sugawara Y, Suzuki M, Tanaka S (2004) Spatial and temporal expression of the ventral pelvic skin aquaporins during metamorphosis of the tree frog, Hyla japonica. J Membr Biol 199:119-126

Hasegawa T, Suzuki M, Tanaka S (2005) Immunocytochemical studies on translocation of phosphorylated aquaporin-h2 protein in granular cells of the frog urinary bladder before and after stimulation with vasotocin. Cell Tissue Res 322:407-415

Heller KB, Lin EC, Wilson TH (1980) The substrate specificity and transport properties of the glycerol facilitator of Escherichia coli. J Bacteriol 144:274-278

Heymann JB, Engel A (2000) Structural clues in the sequences of the aquaporins. J Mol Biol 295:1039-1053

Hibuse T, Maeda N, Funahashi T, Yamamoto K, Nagasawa A, et al. (2005) Aquaporin 7 deficiency is associated with development of obesity through activation of adipose glycerol kinase. Proc Natl Acad Sci U S A 102:10993-10998

Hoffert JD, Leitch V, Agre P, King LS (2000) Hypertonic induction of aquaporin-5 expression through an ERK-dependent pathway. J Biol Chem 275:9070-9077

Holm LM, Jahn TP, Møller AL, Schjoerring JK, Feeri D, et al. (2005) $\mathrm{NH}_{3}$ and $\mathrm{NH}_{4}^{+}$permeability in aquaporin-expressing Xenopus oocytes. Pflug Arch 450:415-428

Ikeda M, Beitz E, Kozono D, Guggino WB, Agre P, et al. (2002) Characterization of aquaporin- 6 as a nitrate channel in mammalian cells. Requirement of pore-lining residue threonine 63. J Biol Chem 277:39873-39879

Ishida N, Hirai SI, Mita S (1997) Immunolocalization of aquaporin homologs in mouse lacrimal glands. Biochem Biophys Res Commun 238:891-895

Ishikawa Y, Skowronshi MT, Inoue N, Ishida H (1998) Alpha(1)adrenoceptor-induced trafficking of aquapoin-5 to the apical plasma membrane of rat parotid cells. Biochem Biophys Res Commun 265:194-200

Ishikawa Y, Iida H, Ishida H (2002) The muscarinic acetylcholine receptor-stimulated increase in aquaporin 5 levels in the apical plasma membrane in rat parotid acinar cells is coupled with activation of nitric oxide/ cGMP signal transduction. Mol Pharmacol 61:1423-1434

Itoh T, Rai T, Kuwahara M, Ko SBH, Uchida S, et al. (2005) Identification of a novel aquaporin AQP12, expressed in pancreatic acinar cells. Biochem Biophys Res Commun 330:832-838

Jung JS, Preston GM, Smith BL, Guggino WB, Agre P (1994) Molecular structure of the water channel through aquaporin CHIP. The hourglass model. J Biol Chem 269:14648-14654

King LS, Nielsen S, Agre P (1996) Aquaporin-1 water channel protein in lung: ontogeny, steroid-induced expression, and distribution in rat. J Clin Invest 97:2183-2191

King LS, Choi M, Fernandez PC, Cartron JP, Agre P (2001) Defective urinary-concentrating ability due to a complete deficiency of aquaporin -1. New Engl J Med 345:175-179

King LS, Nielsen S, Agre P, Brown RH (2002) Decreased pulmonary vascular permeability in aquaporin-1-null humans. Proc Natl Acad Sci USA 99:1059-1063

Kishore BK, Terris JM, Knepper MA (1996) Quantitation of aquaporin-2 abundance in microdissected collecting ducts: Axial distribution and control by AVP. Am J Physiol 271:F62-F70

Knoers NV, Deen PM (2001) Molecular and cellular defects in nephrogenic diabetes insipidus. Pediatr Nephrol 16:1146-1152

Krane CM, Kishore BK (2003) Aquaporins: membrane water channels of the biological world. Biologist 50:81-86

Krane CM, Fortner CN, Hand AR, McGraw DW, Lorenz JN, et al. (2001a) Aquaporin 5-deficient mouse lungs are hyperresponsive to cholinergic stimulation. Proc Natl Acad Sci U S A 98:1411414119

Krane CM, Melvin JE, Nguyen HV, Richardson L, Towne JE, et al. (2001b) Salivary acinar cells from aquaporin 5-deficient mice have decreased membrane water permeability and altered cell volume regulation. J Biol Chem. 276(26):23413-23420

Lachenbruch CA, Diller KR, Pegg DE (1998) Sensitivity of kidney perfusion protocol design to physical and physiological parameters. Ann N Y Acad Sci 858:298-309

Lages F, Lucas C (1995) Characterization of a glycerol/ $\mathrm{H}^{+}$symport in the halotolerant yeast Pichia sorbitolophila. Yeast 11:111-119

Lagree V, Pellerin I, Hubert JF, Tacnet F, Le Caherec F, et al. (1998) A yeast recombinant aquaporin mutant that is not expressed or mistargeted in Xenopus oocyte can be functionally analyzed in reconstituted proteoliposomes. J Biol Chem 273:12422-12426

Lagree V, Froger A, Deschamps S, Hubert JF, Delamarche C, et al. (1999) Switch from an aquaporin to a glycerol channel by two amino acids substitution. J Biol Chem 274:6817-6819

Layne JR, Lee RE, Cutwa MM (1996) Post-hibernation excretion of glucose in urine of the freeze tolerant frog Rana sylvatica. J Herp 30:85-87

Lee RE, Costanzo JP (1993) Integrated physiological responses promoting anuran freeze tolerance. In: Life in the Cold, Carey $\mathrm{C}$, et al. (eds.) (Boulder, CO: Westview Press), pp 501-510

Li C-C, Lin EC (1983) Glycerol transport and phosphorylation by rat hepatocytes. J Cell Physiol 117:230-234

Li J, Verkman AS (2001) Impaired hearing in mice lacking aquaporin-4 water channels. J Biol Chem 276:31233-31237

Liu Z, Shen J, Carbrey JM, Mukhopadhyay R, Agre P, et al. (2002) Arsenite transport by mammalian aquaglyceroporins AQP7 and AQP9. Proc Natl Acad Sci U S A 99: 6053-6058

Lu D, Grayson P, Schulten K (2003) Glycerol conductance and physical asymmetry of the Escherichia coli glycerol facilitator GlpF. Biophys J 85:2977-2987

Lucas C, Costa MD, VanUden N (1990) Osmoregulatory active sodium-glycerol co-transport in the halotolerant yeast Debaryomyces hansenii. Yeast 6:187-192

Ma T, Baoxue Y, Verkman AS (1996) cDNA cloning of a functional water channel from toad urinary bladder epithelium. Am J Phys 271:C1699-C1704

Ma T, Yang B, Gillespie A, Carlson EJ, Epstein CJ, et al. (1997) Generation and phenotype of a transgenic knockout mouse lacking the mercurial-insensitive water channel aquaporin-4. J Clin Invest 100:957-962

Ma T, Yang B, Gillespie A, Carlson EJ, Epstein CJ, et al. (1998) Severely impaired urinary concentrating ability in transgenic mice lacking aquaporin-1 water channels. J Biol Chem 273:4296-4299

Ma T, Song Y, Gillespie A, Carlson EJ, Epstein CJ, Verkman AS (1999) Defective secretion of saliva in transgenic mice lacking aquaporin-5 water channels. J Biol Chem 274:20071-20074

Ma T, Fukuda N, Song Y, Matthay MA, Verkman AS (2000a) Lung fluid transport in aquaporin-5 knockout mice. J Clin Invest 105:93-100

Ma T, Song Y, Yang B, Gillespie A, Carlson EJ, et al. (2000b) Nephrogenic diabetes insipidus in mice lacking aquaporin-3 water channels. Proc Natl Acad Sci U S A 97:4386-4391

Ma T, Hara M, Sougrat R, Verbavatz JM, Verkman AS (2002) Impaired stratum corneum hydration in mice lacking epidermal water channel aquaporin-3. J Biol Chem 277:17147-17153

Manley GT, Fujimura M, Ma T, Noshita N, Filiz F, et al. (2000) Aquaporin-4 deletion in mice reduces brain edema after acute water intoxication and ischemic stroke. Nat Med 6:159-163

Maurel C, Reizer J, Schroeder JI, Chrispeels MJ, Saier MH Jr (1994) Functional characterization of the Escherichia coli glycerol 
facilitator, GlpF,in Xenopus oocytes. J Biol Chem 269:1186911872

Merves M, Bobbitt B, Parker K, Kishore BK, Choo D (2000) Developmental expression of aquaporin 2 in the mouse inner ear. Laryngoscope 110:192-1930

Morishita Y, Sakube Y, Sasaki S, Ishibashi K (2004) Molecular mechanisms and drug development in aquaporin water channel diseases: aquaporin superfamily (superaquaporins): expansion of aquaporins restricted to multicellular organisms. J Pharmacol Sci 96:276-279

Morishita Y, Matsuzaki T, Hara-Chikuma M, Andoo A, Shimono M, et al. (2005) Disruption of aquaporin-11 produces polycystic kidneys following vacuolization of the proximal tubule. Mol Cell Biol 25:7770-7779

Nagai K, Watanabe M, Seto M, Hisatsune A, Miyata T, et al. (2007) Nitric oxide decreases cell surface expression of aquaporin-5 and membrane water permeability in lung epithelial cells. Biochem Biophys Res Commun 354:579-584

Nejsum LN, Kwon TH, Jensen UB, Fumagalli O, Frokiaer J, et al. (2002) Functional requirement of aquaporin-5 in plasma membranes of sweat glands. Proc Natl Acad Sci U S A 99:511-516

Nelson RD, Stricklett P, Gustafson C, Stevens A, Ausiello D, et al. (1998) Expression of an AQP2 Cre recombinase transgene in kidney and male reproductive system of transgenic mice. Am J Physiol 275:C216-C226

Nielsen S, Chou C-L, Marples D, Chirstensen EI, Kishore BK, et al. (1995) Vasopressing increases water permeability of kidney collecting duct by inducing translocation of aquaporin-CD water channels to plasma membrane. Proc Natl Acad Sci U S A 92:1013-1017

Nouwen EJ, Kuhn ER (1983) Radioimmunoassay of arginine vasotocin and mesotocin in serum of the frog Rana ridibunda. Gen Comp Endocrinol 50:242-251

Pang PKT (1983) Evolution of control of epithelial transport in vertebrates. J Exp Biol 106:283-299

Pao GM, Wu LF, Johnson KD, Hofte H, Chrispeels MJ, et al. (1991) Evolution of the MIP family of integral membrane transport proteins. Mol Microbil 5:33-37

Park JH, Saier MH Jr (1996) Phylogenetics characterization of the MIP family of transmembrane channel proteins. J Membr Biol 153:171-180

Pettersson N, Filipsson C, Becit E, Brive L, Hohmann S (2005) Aquaporins in yeasts and filamentous fungi. Biol Cell 97:487500

Preston GM, Agre P (1991) Isolation of a cDNA from erythrocyte integral membrane protein of 28 kilodalton: member of an ancient channel family. Proc Nat Acad Sci U S A 88:11110 11114

Preston GM, Carroll TP, Guggino WB, Agre P (1992) Appearance of water channels in Xenopus oocytes expressing red cell CHIP28 proteins. Science 256:385-387

Preston GM, Jung JS, Guggino WB, Agre P (1993) The mercurysensitive residue at cysteine 189 in the CHIP 28 water channel. J Biol Chem 268:17-20

Rojek A, Fuchtbauer EM, Kwon TH, Frokiaer J, Nielsen S (2006) Severe urinary concentrating defect in renal collecting ductselective AQP2 conditional-knockout mice. Proc Natl Acad Sci U S A 103:6037-6042

Rosenbloom AA, Fisher DA (1974) Radioimmunoassay of arginine vasotocin. Endocrinology 95:1726-1732

Saadoun S, Papadopoulos MC, Hara-Chikuma M, Verkman AS (2005) Impairment of angiogenesis and cell migration by targeted aquaporin-1 gene disruption. Nature 434:786-792

Saparov SM, Liu K, Agre P, Pohl P (2007) Fast and selective ammonia transport by aquaporin-8. J Biol Chem 282:52965301
Schreiber R, Pavenstadt H, Greger R, Kunzelmann K (2000) Aquaporin 3 cloned from Xenopus laevis is regulated by the cystic fibrosis transmembrane conductance regulator. FEBS Lett 475:291-295

Shiels A, Bassnett S (1996) Mutations in the founder of the MIP gene family underlie cataract development in the mouse. Nat Genet 12:212-215

Song Y, Verkman AS (2001) Aquaporin-5 dependent fluid secretion in airway submucosal glands. J Biol Chem 276:41288-41292

Steinfeld S, Cogan E, King LS, Agre P, Kiss R, et al. (2001) Abnormal distribution of aquaporin-5 water channel protein in salivary glands from Sjogren's syndrome patients. Lab Invest 81:143-148

Sui H, Han B-G, Lee JK, Walian P, Jap BK (2001) Structural basis of water-specific transport through the AQP1 water channel. Nature 414:872-878

Suzuki M, Hasegawa T, Ogushi Y, Tanaka S (2007) Amphibian aquaporins and adaptation to terrestrial environments: A review. Comp Biochem Physiol A Mol Integr Physiol (epub ahead of print)

Tajkhorshid E, Nollert P, Jensen MO, Miercke LJ, O'Connell J, et al. (2002) Control of the selectivity of the aquaporin water channel family by global orientational tuning. Science 296:525-530

Tanghe A, Van Dijck P, Dumortier F, Teunissen A, Hohmann S, et al. (2002) Aquaporin expression correlates with freeze tolerance in baker's yeast, and overexpression improves freeze tolerance in industrial strains. Appl Environ Microbiol 6:5981-5989

Tanghe A, van Dijck P, Thevelien JM (2006) Why do microorganisms have aquaporins? Trends Microbiol 14:78-85

Tanii H, Hasegawa T, Hirakawa N, Suzuki M, Tanaka S (2002) Molecular and cellular characterization of a water-channel protein, AQP-h3, specifically expressed in the frog ventral skin. J Membr Biol 188:43-53

Terris J, Ecelbarger CA, Nielsen S, Knepper MA (1996) Long-term regulation of four renal aquaporins in rat. Am $\mathrm{J}$ Physiol 271:F414-F422

Towne JE, Krane CM, Bachurski CJ, Menon AG (2001) Tumor necrosis factor- $\beta$ inhibits aquaporin 5 expression in mouse lung epithelial cells. J Biol Chem 276:18657-18664

Tsubota K (2001) Defective cellular trafficking of lacrimal gland aquaporin-5 in Sjogren's syndrome. Lancet 357:688-689

Uchiyama M (1994) Sites of action of arginine vasotocin in the nephron of the bullfrog kidney. Gen Comp Endocrinol 94:366373

Vom Dahl S, Häussinger D (1997) Evidence for a phloretin-sensitive glycerol transport mechanism in the perfused rat liver. Am J Physiol 272:G563-G574

Virkki LV, Franke C, Somieski P, Boron WF (2002) Cloning and functional characterization of a novel aquaporin from Xenopus laevis oocytes. J Biol Chem 277:40610-40616

Wade JB (1989) Dynamics of apical membrane responses to ADH in amphibian bladder. Am J Physiol 257:R998-R1003

Wade JB, Stetson DL, Lewis SA (1981) ADH action: evidence for a membrane shuttle mechanism. Ann N Y Acad Sci 372:106-117

Yang F, Kawedia JD, Menon AG (2003) Cyclic AMP regulates aquaporin 5 expression at both transcriptional and post-transcriptional levels through a protein kinase A pathway. J Biol Chem 278:32173-32180

Yang B, Song Y, Zhao D, Verkman AS (2005) Phenotype analysis of aquaporin-8 null mice. Am J Physiol Cell Physiol 288:C1161$\mathrm{C} 1170$

Yasui M, Hazama A, Kwon TH, Nielsen S, Guggino WB, et al. (1999) Rapid gating and anion permeability of an intracellular aquaporin. Nature 402:184-187

Zardoya R (2005) Phylogeny and evolution of the major intrinsic protein family. Biol Cell 97:397-414 
Zeidel ML, Ambudkar SV, Smith BL, Agre P (1992) Reconstitution of functional water channels in liposomes containing purified red cell CHIP28 protein. Biochemistry 31:7436-7440

Zhang R, van Hoek AN, Biwersi J, Verkman AS (1993) A point mutation at cysteine 189 blocks the water permeability of rat kidney water channel CHIP28k. Biochemistry 32:2938-2941
Zimmerman SL, Frisbie J, Goldstein DL, West J, Rivera K, et al. (2007) Excretion and conservation of glycerol, and expression of aquaporins and glyceroporins, during cold acclimation in Cope's gray tree frog Hyla chrysoscelis. Am J Physiol Regul Integr Comp Physiol 292:R544-R555 